\title{
Emergencia de variante Delta- B.1.617.2. Su impacto potencial en la evolución de la pandemia por SARS-CoV-2
}

\author{
Delta-B.1.617.2 variant emergency. Its potential impact on the evolution of \\ the SARS-CoV-2 pandemic
}

\begin{abstract}
Dr. Eduardo López Mora ${ }^{1^{*}}$
Dr. Jorge Espinoza Rojas ${ }^{2}$

Dra. Jeannette Dabanch Peña ${ }^{3,5}$

Dr. Rodrigo Cruz Choappa ${ }^{4,5}$

${ }^{1}$ Residente Infectología Universidad de Valparaíso. Hospital San Camilo de San Felipe. ${ }^{2}$ Residente Infectología Universidad de Valparaíso. Hospital Gustavo Fricke de Viña del Mar.

${ }^{3}$ Hospital Clínico de la Universidad de Chile

${ }^{4}$ Hospital Clínico IST y Hospital de Quilpué.

${ }^{5}$ Centro de Diagnóstico e Investigación de Enfermedades Infecciosas (CDIEI)

Universidad de Valparaíso

*Autor para correspondencia: eduardo.lopez@live.cl
\end{abstract}

RECIBIDO: 4 de julio 2021

APROBADO: 6 de julio 2021

DOI: 10.22370/bolmicol.2021.36.1.2883

Palabras claves: SARS-CoV-2, variante Delta.

Keywords: SARS-CoV-2, Delta variant.

\section{RESUMEN}

Se describe hasta la fecha de hoy, 4 de julio del 2021, la evidencia existente sobre la variante Delta del SARS-CoV-2, su impacto en la trasmisión, en la severidad de la infección y su probable evasión a la respuesta inmune.

\footnotetext{
ABSTRACT

As of today, July 4, 2021, the existing evidence on the Delta variant of SARS-CoV-2, its impact on transmission, on the severity of the infection
}

and its probable evasion of the immune response is described.

\section{INTRODUCCIÓN}

Al 1 de julio del 2021 el virus SARS-CoV-2 ha causado más de 180 millones de infecciones y 3,9 millones de muertes (1). La OMS ha aprobado el uso de emergencia de 7 vacunas, que provienen de 3 plataformas distintas, sin embargo, hay más de 100 vacunas en fase de desarrollo clínico (2). Se ha impulsado una campaña de vacunación global que a la fecha ha significado la administración de cerca de 3 
Emergencia de variante Delta- B.1.617.2. Su impacto potencial en la evolución de la pandemia - López E.

billones de dosis de vacunas y se estima que cerca del $24 \%$ de la población mundial ha recibido al menos 1 dosis. En Chile, se ha llegado a cerca de 11 millones de personas con esquema completo (3).

Los virus ARN pueden acumular una mayor cantidad de mutaciones, a través del proceso de selección natural y las mutaciones neutrales o beneficiosas pueden transmitirse de generación en generación. El elevado número de casos en distintas partes del mundo y los viajes internacionales han permitido la emergencia y posterior diseminación de variantes (4).

El impacto de la emergencia de estas nuevas variantes dependerá de su potencialidad en el aumento de la transmisión, asociación a enfermedad más grave (aumento de hospitalizaciones o muertes), evasión de la respuesta inmune posterior a una infección o vacunación previa y/o posibles fallas en la detección diagnóstica que signifiquen en una pérdida de la efectividad de las medidas de contención y un impacto grave en la respuesta de los sistemas de salud. Estas características han llevado a clasificar a las distintas variantes en aquellas de preocupación o interés. A partir del 31 de mayo de 2021, la OMS propuso etiquetas para las variantes de preocupación e interés, de las cuales se mantiene permanente actualización según la evolución de la pandemia (5).

\section{Situación en Chile}

En Chile, durante el mes de junio se han secuenciado 459 muestras de las cuales se identificaron 344 casos de variantes de preocupación $(74,9 \%)$; la variante Gamma fue identificada en 334 muestras, y 9 muestras para la variable Alfa. Cabe mencionar que el 24 de junio se confirmó el primer caso de variable Delta en el país. En relación con las variables de interés hubo 85 casos de los cuales 82 de ellos correspondieron a la variable Lambda (6).

\section{Variante delta (VOC.B.1.617.2)}

Identificada inicialmente en India en octubre del 2020, pasó a formar parte de variantes de interés el 4 de abril del 2021 y a ser considerada como variante de preocupación a partir del 11 de mayo del 2021 (7).

Ha sido descrita en 84 países, en la región de las Américas se ha reportado en EE UU (52 estados), Canadá, México, Brasil, Argentina, Perú y Chile (Figura 1). Según los datos provenientes de GISAID, al 4 de julio de 2021, se han detectado más de 111 mil muestras secuenciadas de esta variante en todo el mundo (8).

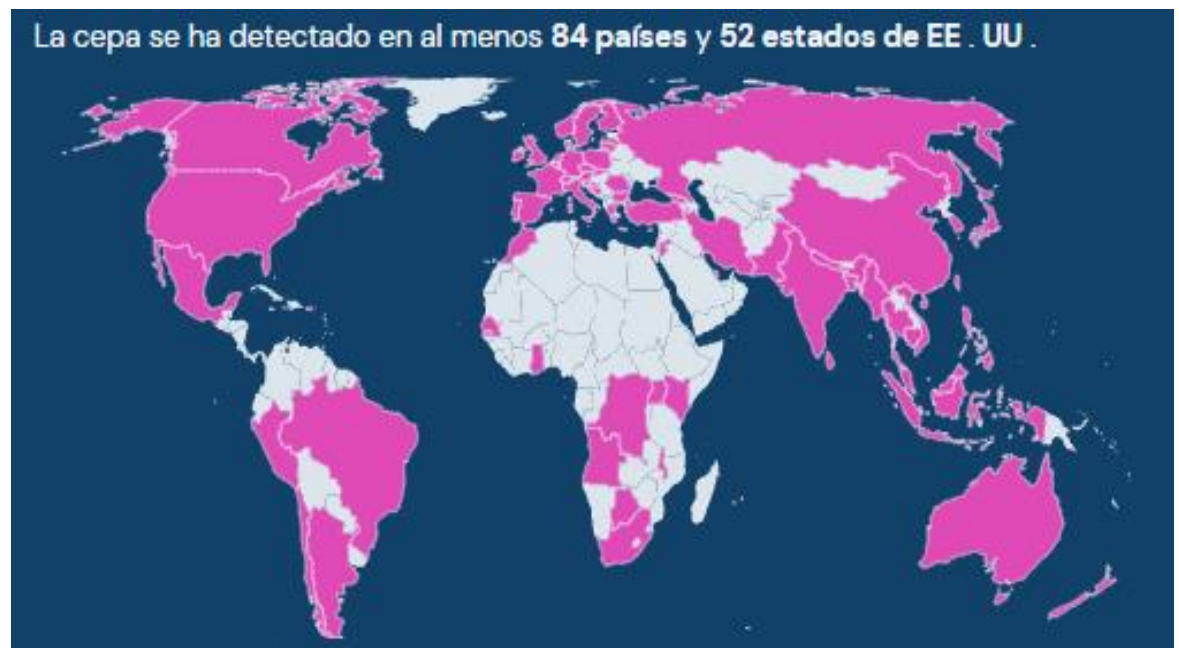

Figura 1. https://outbreak.info/ 
Emergencia de variante Delta- B.1.617.2. Su impacto potencial en la evolución de la pandemia - López E.

Esta variante consta de más de 15 mutaciones, siendo las de mayor relevancia aquellas que comprometen a la proteína Spike: T19R, L452R, E484Q, T478K, P681R, D950N, de éstas E484Q y la L452R en el dominio de unión a receptor (RBD), motivo de su apodo coloquial de "doble mutante" (Figura 2) (9).

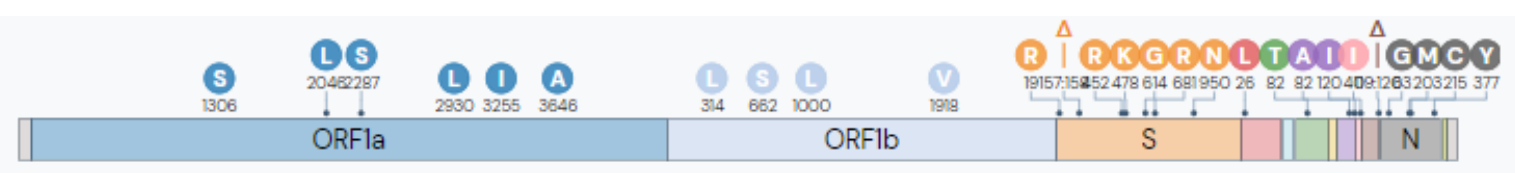

Figura 2. https://outbreak.info/

Según la evidencia disponible a la fecha, la variante de SARS-CoV-2 Delta (B.1.617.2) es un 40-60\% más transmisible que la variante Alfa (B.1.1.7) (10). También puede estar asociada con mayor riesgo de hospitalización (11). Además, existe evidencia de que en aquellas personas que solo han recibido esquema parcial de vacunación estarían menos protegidos contra esta variante que contra otras, independiente de la plataforma utilizada (mRNA o vector viral) (12).

\section{Variante Delta y mutación K417N}

En las últimas semanas se han identificado dos sublinajes de la variante Delta que destacan por la mutación K417N en la proteína Spike ("Delta plus"), las cuales han sido denominadas AY.1 (B.1.617.2.1) e AY.2 (B.1.617.2.2), descritas por primera vez en abril y marzo de este año, respectivamente, con una propagación por ahora limitada, acumulando a la fecha cada una poco más de 200 secuenciaciones en la base de datos GISAID (Figura 3) (14). Se debe mencionar que esta mutación K417N es compartida con otros sublinajes de la variante Beta (B.1.351), la cual ha demostrado por un lado una menor afinidad entre el dominio de unión a receptor (RBD) con ACE2, como también una menor afinidad con anticuerpos neutralizantes, evadiendo así la respuesta inmune. Cabe señalar a lo anterior que probablemente esta menor afinidad por el RBD, se compensa con las mutaciones propias de la variante Delta en el RBD que permiten una mayor afinidad con el receptor, optimizando así su transmisión (15).

Sobre la base de la ventaja de transmisión estimada en esta variante se proyecta que el $70 \%$ de las nuevas infecciones por SARS-CoV-2 se deberán a esta variante en Europa a principios de agosto y el $90 \%$ de las infecciones a finales de agosto. A lo anterior sin embargo se debe considerar que el riesgo para poblaciones completamente vacunadas es bajo y muy alto para aquellas poblaciones no vacunadas. El potencial impacto debe considerar el universo de población completamente vacunada y el efecto de medidas de contención de salud pública (13).

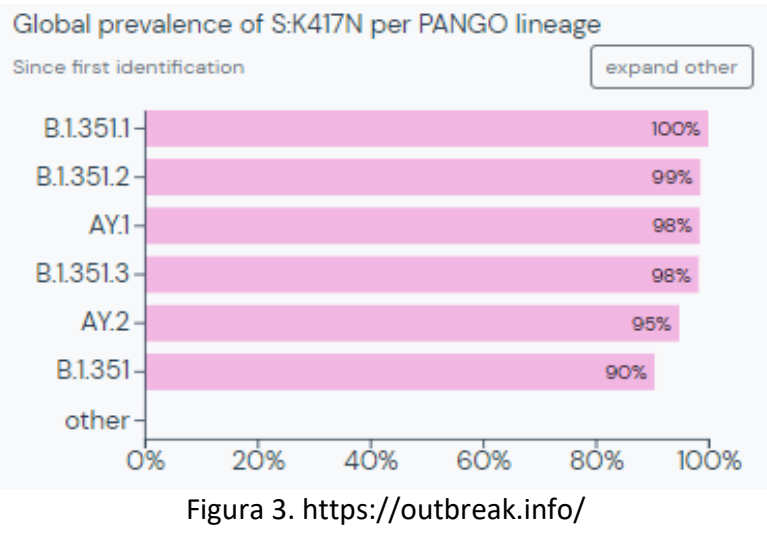

\section{DISCUSIÓN}

La evolución de la pandemia por SARS-CoV-2 ha sido marcada por la emergencia de variantes, 
Emergencia de variante Delta- B.1.617.2. Su impacto potencial en la evolución de la pandemia - López E.

las cuales, dado su impacto en potenciar una mayor transmisión, evasión del sistema inmune y severidad, han sido clasificadas por la OMS, de acuerdo al peligro que revisten para la respuesta de los sistemas de salud $(1,2)$. La emergencia de la variable Delta, de la cual ya hay datos que la relacionan a mayor transmisión, menor efecto de neutralización por anticuerpos y más hospitalizaciones, reviste una grave amenaza $(10,11)$. Por otra parte, la vacunación, independiente de la plataforma, ha demostrado ser efectiva y eficaz en disminuir la transmisión, los casos sintomáticos y la mortalidad asociada, también para la variable Delta $(11,12,13)$.

La respuesta inmune adaptativa es clave en el control exitoso de la infección por SARS-CoV2 , tanto la respuesta celular como la humoral (14). Las vacunas son un factor clave en contener el efecto de la emergencia de variantes, siempre y cuando se logre cortar la cadena de transmisión y disminuir los casos a nivel global, factor más relevante en la emergencia de variantes (15). Cabe señalar que, si bien a la fecha cerca de un cuarto de la población mundial se encuentra vacunada, hay sendas diferencias en acceso y oportunidad, principalmente debido al nivel de desarrollo de los distintos países. El riesgo de emergencia de variantes debe ser manejado de acuerdo a metas globales.

En lo inminente, el vacunar con prontitud, con esquema completo, especialmente a la población con factores de riesgo, parece ser clave en la respuesta a corto plazo, como también la necesidad de sostener las medidas de prevención y una adecuada comunicación del riesgo a la población.

Conflictos de intereses: los autores declaran no tener conflictos de intereses.

Financiamiento: Universidad de Valparaíso.

\section{REFERENCIAS}

1. WHO Coronavirus Dashboard. June https://covid19.who.int

(COVID-19) 2021.

2. WHO. COVID-19 vaccine tracker and landscape.

https://www.who.int/publications/m/ite $\underline{\mathrm{m} / \text { draft-landscape-of-covid-19- }}$ candidate-vaccines

3. Coronavirus (COVID-19) Vaccinations. https://ourworldindata.org/covidvaccinations

4. R. Cruz, E. López. SARS-CoV-2: La emergencia de variantes y su impacto en el control de la pandemia. Bol. Micol. 2021; 36(1):1-3

5. WHO. Tracking SARS-CoV-2 variants. https://www.who.int/en/activities/tracki ng-SARS-CoV-2-variants/

6. Reporte de circulación de variantes SARS-CoV-2 en Chile 25 de junio. https://www.minsal.cl/wpcontent/uploads/2021/07/Informe-devariantes-FIN.pdf

7. Comunicado SOCHINF. Variante Delta. https://sochinf.cl/comunicado-sochinfsars-cov-2-variante-delta-en-chile. Julio 2021.

8. B.1.617.2 Lineage Report. Outbreak. 24 Julio 2021. https://outbreak.info/situation-reports.

9. Pango Linages. (https://cov-
lineages.org/global_report.html) 
Emergencia de variante Delta- B.1.617.2. Su impacto potencial en la evolución de la pandemia - López E.

10. Public Health England. SARS-CoV-2 variants of concern and variants under investigation in England Technical briefing $12 \quad 2021$. (https://assets.publishing.service.gov.uk/ government/uploads/system/uploads/att achment_data/file/988619/Variants_of_ Concern_VOC_Technical_Briefing_12_ England.pdf)

11. Sheikh A, McMenamin J, Taylor B, Robertson C. SARS-CoV-2 Delta VOC in Scotland: demographics, risk of hospital admission, and vaccine effectiveness. The Lancet. 2021 Jun 26.

12. López J, Andrews N, Gower C, Gallagher E, Simmons R, Thelwall S, Stowe J, et al. Effectiveness of COVID19 vaccines against the B.1.617.2 variant. May 2021. medRxiv 2021.05.22.21257658. Preprint.

13. Threat Assessment Brief: Implications for the EU/EEA on the spread of the SARS-CoV-2 Delta (B.1.617.2) variant of concern

(https://www.ecdc.europa.eu/en/publicat ions-data/threat-assessment-emergenceand-impact-sars-cov-2-delta-variant. 23 June)

14. Mullen J, Tsueng G, Latif A, Alkuzweny M, Cano M, Haag E, et al and the Center for Viral Systems Biology outbreak.info. Available online: https://outbreak.info/ (2020)

15. Fratev F. The N501Y and K417N mutations in the spike protein of SARSCoV-2 alter the interactions with both hACE2 and human derived antibody: A Free energy of perturbation study. bioRxiv 2020.12.23.424283. 